\title{
PARAMETER ESTIMATION OF PARABOLIC TYPE FACTOR MODEL AND EMPIRICAL STUDY OF US TREASURY BONDS
}

\author{
S.I. Aihara, ${ }^{1}$ and A. Bagchi, ${ }^{2}$ \\ ${ }^{1}$ Tokyo University of Science, Suwa Toyohira,5000-1 Chino, Nagano, Japan, aihara@rs.suwa.tus.ac.jp \\ ${ }^{2}$ FELab and Department of Applied Mathematics, University of Twente, P.O.Box 217, 7500AE \\ Enschede,The Netherlands, a.bagchi@ewi.utwente.nl
}

\begin{abstract}
In this paper we study the parameter estimation problem for stochastic distributed parameter systems by using the modified maximum likelihood method. More specifically, by using the US treasury bond data, the parameter estimation is performed for the stochastic hyperbolic and parabolic models describing the behavior of the term-structure of the US bond. From the prediction results, we can show that the parabolic factor models work better than the hyperbolic ones.
\end{abstract}

Key words: Factor model, US bonds, MLE, Stochastic Parabolic Equation, Maximum likelihood estimate

\section{Introduction}

Parameter estimation problem for stochastic distributed parameters has a long history and there still exist many open problems. In this paper, we present a practical application of the parameter estimation to a financial engineering problem. Let $P(t, T)$ denote the bond price where $t$ is a present time and $T$ denotes the maturity. The bond price $P(t, T)$ changes randomly in value and at $t=T P(T, T)$ takes the preassigned value.

From the relation that $P(t, T)=\exp \left\{-\int^{T-t} f(t, x) d x\right\}$, the forward rate process $f(t, x)$ may be directly modeled instead of $P$. In this paper, we check the feasibility of the model selection of forward rate process by using some real data.

Here we use the treasury bills data which are easily obtained from the website. In US government securities, we used the constant maturity bond data,i.e., 1 year (starting date 01/02/1962) 2 year (starting date 06/01/1976) 3 year (starting date 01/02/1962) 5 year (starting date 06/01/1962) 7 year (starting

Please use the following format when citing this chapter:

Author(s) [insert Last name, First-name initial(s)], 2006, in IFIP International Federation for Information Processing, Volume 199, System Modeling and Optimization, eds. Ceragioli F., Dontchev A., Furuta H., Marti K., Pandolfi L., (Boston: Springer), pp. [insert page numbers]. 
date $07 / 01 / 1964$ ) 10 year (starting date 01/02/1962) 20 year (starting date 10/01/1993) .

Noting that 20-year bond only starts at 10/01/1993, we cut past date for other bonds and set all data which start from this date up to 05/28/2004. In Fed data, there are missing parts and so we adjust these data by using the method proposed by Cochrane.(See http://gbs.uchikago.edu/fac /john.cochrane/)

To derive the forward rate process, the obtained yield data are regarded as zero-coupon curve. Hence we have the following relation between forward rate $f(t, x)$ and the yield curve $Y(t, T)$ such that

$$
\frac{1}{T-t} \int_{0}^{T-t} f(t, x) d x=\log (1+Y(t, T)) .
$$

Theoretically speaking, if we differentiate the above equation with respect to $T-t$, we can get the forward rate process $f(t, x)$. However, we only obtain 7 different maturity bonds. Firstly, we use the usual curve fitting procedure as stated in [1] and next we differentiate this process with respect to $T-t$ and obtain the forward rate process. As was mentioned in [1], the obtained results strongly depend on the methods used. For example, if we use the cubic spline and differentiate the interpolated process, the obtained forward process is largely volatile at the long maturity part. To aviod this we use the interpolation with cubic-function which is found in MATLAB as 'interp 1(..., 'cubic').m'.

In Fig.1, you can see the original T-bond yield curves. By using the cubic interpolation ( interp1 with 'cubic' in MATLAB) we obtain the smooth yield curve and differentiate this process. In Fig.1, the derived forward rate process is demonstrated. Now from this process, we shall try to identify parameters contained in the dynamics. Here we use the classical procedure to identify the several parameter functions. The main aim of this paper is to show that the parabolic type dynamics is experimentally accepted as the forward rate dynamics.

\section{Hyperbolic system modeling}

The most popular dynamics of the forward rate processes is a hyperbolic type partial differential equation which was first introduced by Heath, Jarrow and Morton from the absence of arbitrage argument and developed further by Santa-Clara et. al [2] and Aihara and Bagchi [3].

The general hyperbolic model is given by

$$
\begin{aligned}
d f(t, x) & =\frac{\partial f(t, x)}{\partial x} d t+\mu(x) d t+d w(t, x) \\
f(0, x) & =f_{o}(x)
\end{aligned}
$$




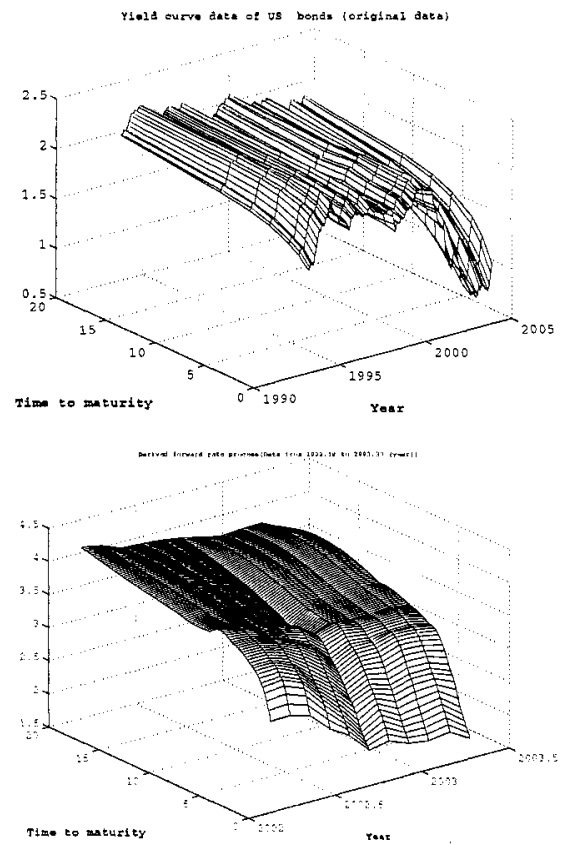

Figure 1. Original yield curve data (US bonds) and derived forward rate

where

$$
E\{w(t, x) w(t, y)\}=q(x, y) t .
$$

We need the following regularity property for $f(t, x)$ to perform the parameter identification.

THEOREM 1 We assume that

$$
f_{0} \in L^{2}\left(\Omega ; H^{m}(\tilde{G})\right), \mu \in H^{m}(\tilde{G})
$$

and

$$
\operatorname{Tr}\left\{\frac{\partial^{m}}{\partial x^{m}}\left(\frac{\partial^{m}}{\partial x^{m}} Q\right)^{*}\right\}<\infty,
$$

where $\tilde{G}=] 0, \hat{T}+t_{f}[$ with $G=] 0, \hat{T}\left[H^{m}\right.$ denotes the $m$-th order Sobolev space and $Q=\int_{\tilde{G}} q(x, y)(\cdot) d y$. Then

$$
f \in L^{2}\left(\Omega ; C\left(\bar{T}_{f} ; H^{m}(G)\right),\right.
$$

where $\left.T_{f}=\right] 0, t_{f}[$

The proof can be found in [3]. 


\subsection{Identification of the covariance kernel}

The most important part of the forward model is to identify the covariance kernel of the noise process. To estimate this kernel, we use the classical procedure by using some properties of the Ito stochastic integral. Noting that

$$
E\{(w(t), w(t))\}=\operatorname{Tr}\{Q\} t
$$

we have

$$
\operatorname{Tr}\{Q\} t=|f(t)|^{2}-|f(0)|^{2}-2 \int_{0}^{t}(f(s), d f(s)),
$$

where $(\cdot, \cdot)$ and $|\cdot|$ denote the inner product and norm in $L^{2}(\tilde{G})$. The discreteversion of the formula (6) is

$$
\begin{aligned}
\sum\left(f\left(t_{i+1}, x\right)-f\left(t_{i}, x\right)\right)\left(f\left(t_{i+1}, y\right)\right. & \left.-f\left(t_{i}, y\right)\right) \\
& \sim q(x, y) t .
\end{aligned}
$$

Applying (6) to T-bond data, the estimated kernel of $q(x, y)$ is shown in Fig.2. Here we used the data $f(t, x)$ for $2000.64 \leq t$ (year) $\leq 2002.183$ shown in Fig. 1 . In the obtained results, the value of the kernel at the long maturity parts
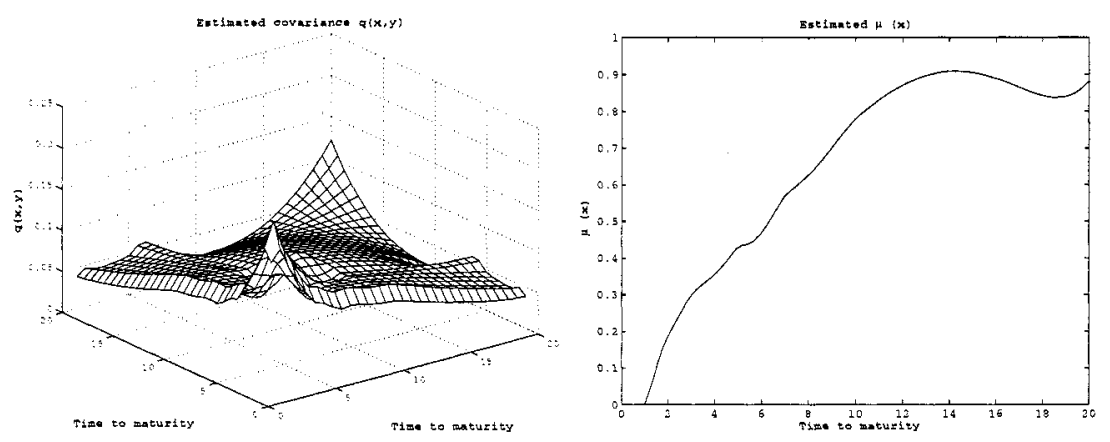

Figure 2. Estimated kernel $q(x, y)$ and $\int_{0}^{x} q(x, y) d y$

seems to be rather big. This phenomena may be caused by the interpolation method "cubic-function".

(i) Modeling in the risk neutral world

Hereafter we set the kernel $q(x, y)$ as the estimated one. In the risk neutral world, the function $\mu(x)$ is set as

$$
\mu(x)=\int_{0}^{x} q(x, y) d y .
$$


Hence the $\mu(x)$-process becomes as shown in Fig.2.

In order to check the feasibility of this model, we simulated the hyperbolic equation, setting the value of the initial condition as $f(2002.183,:)$. Without adding the noise $w(t, x)$, we obtain $E\left\{f(t, x) \mid f_{o}=f(2002.183,:)\right\}$. In Fig.3, the predicted value is shown.

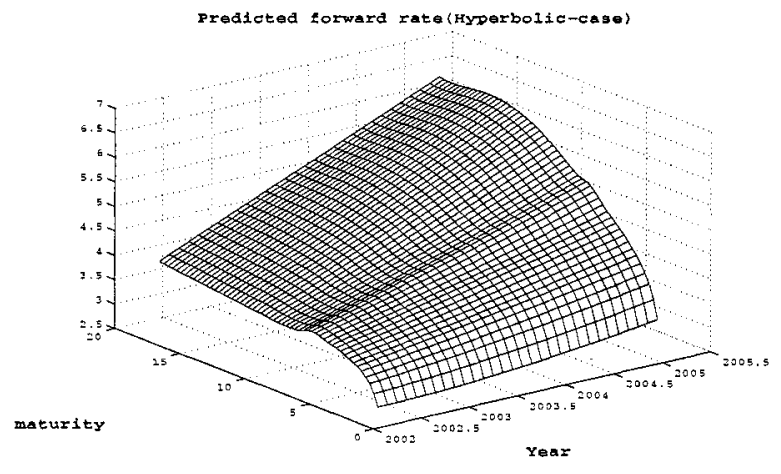

Figure 3. Predicted forward rate(Hyperbolic case)

From this result, we clearly see that our observed data is not in the risk neutral world. So we need to identify the market price of risk in the next subsection.

(ii) Identification of market price of risk:

Here we consider the following restriction: The market price of risk has a form;

$$
\lambda \sqrt{q(x, x)}
$$

i.e, we reset $\mu(x)$ as

$$
\mu(x)=\lambda \sqrt{q(x, x)}+\int_{0}^{x} q(x, y) d y
$$

This $\lambda$ has primarily been invented to price consistently interest rate derivatives rather than to fit the historical evolution of the forward rate process. However, this parameter $\lambda$ is still needed to reproduce the forward rate process.

It is interesting that the parameter $\lambda$ of market price of risk may be identified to maximize the modified log likelihood functional. The used data are the same as those used in the previous identification.

The exact likelihood function for an infinite-dimensional system is difficult to derive without any strict conditions. However, from Thorem 1, we can define the modified likelihood functional by setting $m=1$ and get $f \in$ $L^{2}\left(\Omega ; C\left(\bar{T}_{f} ; H^{1}\right)\right)$. Hence

$$
M L F=\int_{t_{1}}^{t_{2}}\left(\frac{\partial f(s, x)}{\partial x}+\lambda \sqrt{q(x, x)}\right.
$$




$$
\begin{array}{r}
\left.+\int_{0}^{x} q(x, y) d y, d f(s, x)\right) \\
-\frac{1}{2} \int_{t_{1}}^{t_{2}}\left|\frac{\partial f(s, x)}{\partial x}+\lambda \sqrt{q(x, x)}+\int_{0}^{x} q(x, y) d y\right|^{2} d s,
\end{array}
$$

where $t_{2}=2002.185, t_{1}=2000.646$ and

$$
\left(\phi_{1}, \phi_{2}\right)=\int_{0}^{19} \phi_{1}(x) \phi_{2}(x) d x,|\phi|^{2}=(\phi, \phi) .
$$

To derive the exact likelihood functional we need to support the invertibility of the covariance kernel

$$
Q(\cdot)=\int_{0}^{19} q(x, y)(\cdot) d y
$$

In the infinite dimensional case, the operator $Q$ is not invertible. Ultimately we replace the weight $Q$ appearing in the likelihood functional by the identity operator. We call this the modified likelihood functional. To avoid this ambiguity, using the principal component analysis, we can pick up finite principal components. In such a case, we can derive the inverse of $Q$ and the exact likelihood can be derived. However, the proposed modified likelihood is easily constructed without using principal component analysis and still contains the infinite number of random sources. The maximum MLE $\hat{\lambda}$ is given by

$$
\begin{gathered}
\hat{\lambda}=\left[\int_{t_{1}}^{t_{2}}(\sqrt{q(x, x)}, d f(t, x))\right. \\
\left.-\int_{t_{1}}^{t_{2}}\left(\sqrt{q(x, x)}, \frac{\partial f(t, x)}{\partial x}+\mu(x)\right) d s\right] / \operatorname{Tr} Q\left(t_{2}-t_{1}\right) .
\end{gathered}
$$

The derived $\hat{\lambda}$ and the predicted forward rate process are respectively shown in Fig.4.

In Fig.5, we present the real forward rate and predicted processes, respectively.

\section{Parabolic modeling}

In this section, we introduce the parabolic type partial differential equation for the forward rate process instead of the hyperbolic type. This model was already proposed by Bouchaud et.al [4] and [5] to support the smoothness of the forward process with respect to time-to-maturity and that the information diffuses from one maturity to the next.

In the empirical studies, we find that the adjusting term is needed to fit the historical data. In addition to the $\lambda \sqrt{q(x, x)}$ term, we add the diffusion term in the model, because from Fig. 5 it seems that the shape of the real forward rate process is a diffused shape rather than the predicted shape from the 

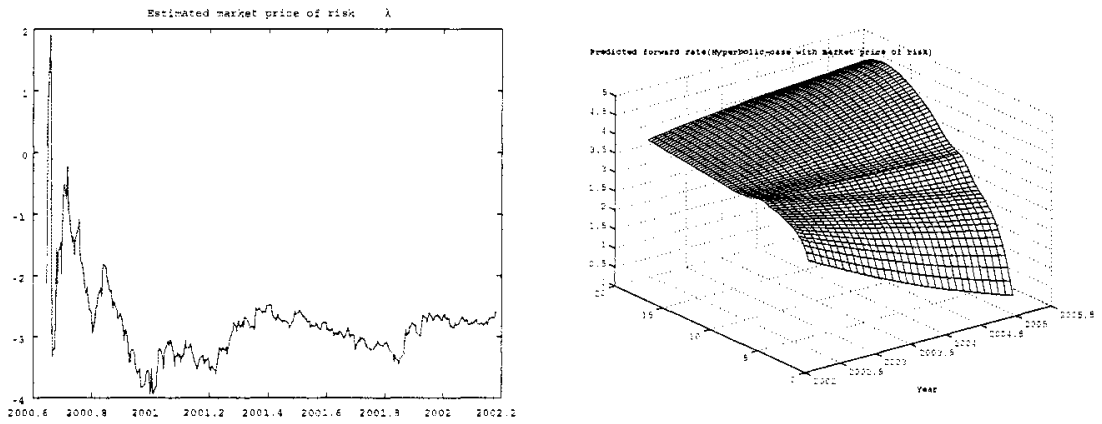

Figure 4. Estimated market price of risk and predicted forward rate

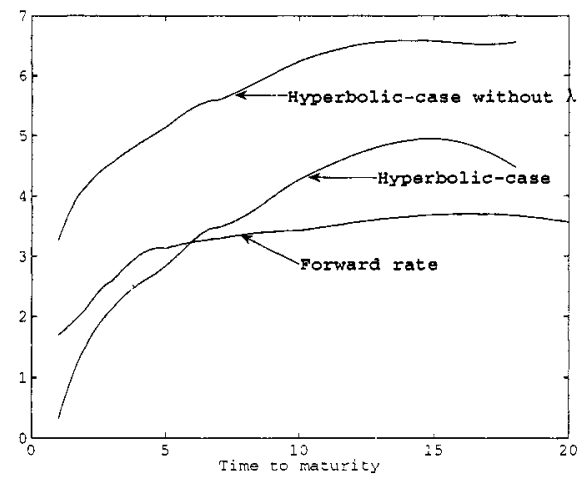

Figure 5. Predicted forward rates and real rate

hyperbolic model. Hence we set the simple parabolic type equation for the forward rate model and identify the systems parameters from the data used from $1999.5 \leq t \leq 2001.79$. From this experiment, we can conclude that the parabolic modeling is more efficient than the hyperbolic modeling.

We consider the following model: for $x \in G=] 0,19[$,

$$
\begin{aligned}
d f(t, x) & =k \frac{\partial^{2} f(t, x)}{\partial x^{2}} d t+\frac{\partial f(t, x)}{\partial x}+\mu(x) d t+d w(t, x) \\
f(0, x) & =f_{0}(x) \\
\frac{\partial f(t, 0)}{\partial x} & +\alpha_{1} f(t, 0)=\beta_{1}, \frac{\partial f(t, 19)}{\partial x}+\alpha_{2} f(t, 19)=\beta_{2} .
\end{aligned}
$$

We work in the following Hilbert spaces:

$$
V=H^{1}(G) \subset H=L^{2}(G) \subset V^{\prime}=\text { dual of } V .
$$


Define

$$
\begin{array}{r}
<A \phi_{1}, \phi_{2}>=\int_{G}\left\{k \frac{\partial \phi_{1}}{\partial x} \frac{\partial \phi_{2}}{\partial x}-\frac{\partial \phi_{1}}{\partial x} \phi_{2}\right\} d x \\
+k \alpha_{2} \phi_{1}(19) \phi_{2}(19)-k \alpha_{1} \phi_{1}(0) \phi_{2}(0), \forall \phi_{1}, \phi_{2} \in V
\end{array}
$$

The variational form of the system becomes $\forall \phi \in V$,

$$
(f(t), \phi)+\int_{0}^{t}<A f(s), \phi>d s=\left(f_{o}, \phi\right)+\int_{0}^{t}(\mu, \phi) d s+(w(t), \phi) .
$$

THEOREM 2 We assume

(C-1) $k>0$

(C-2) $f_{0} \in L^{2}(\Omega ; H), \mu \in L^{2}\left(\Omega \times T_{f} ; V^{\prime}\right)$

(C-3) $\operatorname{Tr}\{Q\}<\infty$. (15) has a unique solution in

$$
L^{2}\left(\Omega ; C\left(\bar{T}_{f} ; H\right) \cap L^{2}\left(T_{f} ; V\right)\right) .
$$

Proof. The parabolic type stochastic evolution equations have been studied by many authors,e.g., [6],[7].

In order to define the modified likelihood functional we need the following theorem:

THEOREM 3 In addition to all conditions of Theorem 3.1, we set

(C-4) $f_{0} \in L^{2}(\Omega ; V), \mu \in L^{2}\left(\Omega \times T_{f} ; H\right)$

(C-5) $\operatorname{Tr}\left\{\frac{\partial}{\partial x}\left(\frac{\partial Q}{\partial x}\right)^{*}\right\}<\infty$

Hence we have

$$
f \in L^{2}\left(\Omega ; C\left(\bar{T}_{f} ; V\right) \cap L^{2}\left(T_{f} ; V \cap H^{2}\right)\right) .
$$

By using the similar method used in [3] we can prove this theorem.

We can use the same technique for identifying $q(x, y)$ in section 2 . Furthermore we also set

$$
\begin{array}{r}
\mu(x)=\lambda \sqrt{q(x, x)}+\tilde{\mu}(x) \\
\tilde{\mu}(x)=\int_{0}^{x} q(x, y) d y .
\end{array}
$$

We need to identify unknown parameters $k, \alpha_{1}, \alpha_{2}, \beta_{1}, \beta_{2}$ and $\lambda$.

(i) Identification of boundary parameters:

Noting that from Theorem $3, \frac{\partial f(t, 19)}{\partial x}$ and $\frac{\partial f(t, 0)}{\partial x}$ belong to $L^{2}\left(\Omega \times T_{f} ; R^{1}\right)$, respectively, we can apply the usual least square method and obtain the following algorithm:

$$
\begin{array}{r}
\hat{\alpha}_{1}=\frac{\int_{t_{1}}^{t_{2}}\left(\frac{\partial f(s, 0)}{\partial x}-\frac{\partial f\left(t_{1}, 0\right)}{\partial x}\right)\left(f(s, 0)-f\left(t_{1}, 0\right)\right) d s}{\int_{t_{1}}^{t_{2}}\left(f(s, 0)-f\left(t_{1}, 0\right)\right)^{2} d s} \\
\hat{\beta}_{1}=\frac{\int_{t_{2}}^{t_{2}} \frac{\partial f(s, 0)}{\partial x} d s+\int_{t_{1}}^{t_{2}} \hat{\alpha}(s) f(s, 0) d s}{t_{2}-t_{1}},
\end{array}
$$


and for the boundary $x=19$ we can set the similar algorithm.

The estimated results are shown in Fig.6 for $1999.5 \leq t_{2} \leq 2002$.
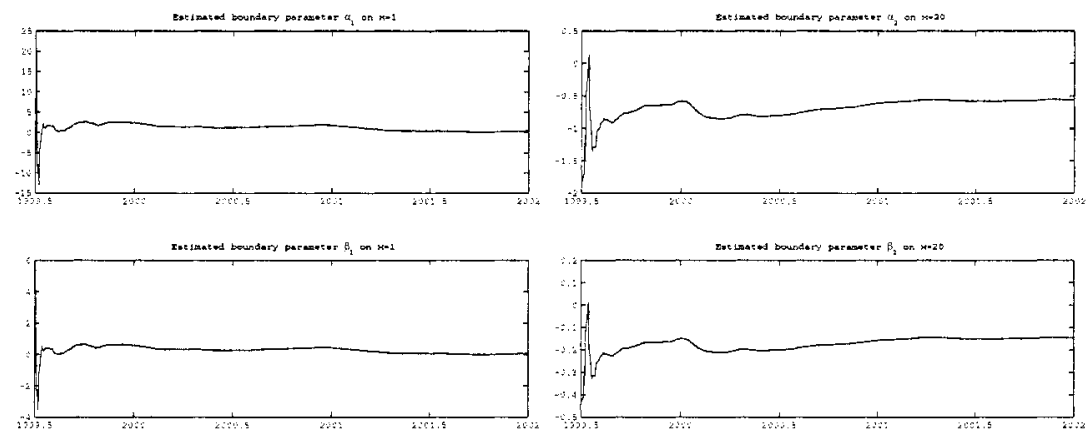

Figure 6. Sample runs of estimated boundary parameters

(ii) Identification of $k$ and $\lambda$ :

In order to identify the diffusion coefficient $k$ and $\lambda$, we also introduced the modified likelihood functional:

$$
\begin{aligned}
M L F= & \int_{t_{1}}^{t_{2}}\left(k \frac{\partial^{2} f(t, x)}{\partial x^{2}}+\frac{\partial f(t, x)}{\partial x}+\tilde{\mu}(x)+\lambda \sqrt{q(x, x)}, d f(t, x)\right) \\
& -\frac{1}{2} \int_{t_{1}}^{t_{2}}\left|k \frac{\partial^{2} f(t, x)}{\partial x^{2}}+\frac{\partial f(t, x)}{\partial x}+\tilde{\mu}(x)+\lambda \sqrt{q(x, x)}\right|^{2} d s,
\end{aligned}
$$

where we already find that $f \in L^{2}\left(\Omega \times T_{f} ; H^{2}(G)\right)$ from 2. The maximum MLF $\hat{k}$ and $\hat{\lambda}$ are given by

$$
\left[\begin{array}{l}
\hat{k} \\
\hat{\lambda}
\end{array}\right]=M^{-1} N,
$$

where

$$
\begin{gathered}
M=\left[\begin{array}{cc}
\int_{t_{1}}^{t}\left|\frac{\partial^{2} f(s, x)}{\partial x^{2}}\right|^{2} d s & \int_{t_{1}}^{t}\left(\frac{\partial^{2} f(s, x)}{\partial x^{2}}, \sqrt{q(x, x)}\right) d s \\
\int_{t_{1}}^{t}\left(\frac{\partial^{2} f(s, x)}{\partial x^{2}}, \sqrt{q(x, x)}\right) d s & \int_{t_{1}}^{t}(\sqrt{q(x, x)}, \sqrt{q(x, x)}) d s
\end{array}\right] \\
N=\left[\begin{array}{c}
\int_{t_{1}}^{t}\left(\frac{\partial^{2} f(s, x)}{\partial x^{2}}, d f(s, x)\right)-\int_{t_{1}}^{t}\left(\frac{\partial f(t, x)}{\partial x}+\hat{\mu}(x), \frac{\partial^{2} f(s, x)}{\left.\partial x^{2}\right)}\right) d s \\
\int_{t_{1}}^{t}(\sqrt{q(x, x)}, d f(s, x))-\int_{t_{1}}^{t}\left(\frac{\partial f(t, x)}{\partial x}+\hat{\mu}(x), \sqrt{q(x, x)}\right) d s
\end{array}\right] .
\end{gathered}
$$

The sample runs of the estimated $\lambda$ and $k$ are shown in Fig.7. The predicted value of forward rate is given in Fig. 8 with its true value. 

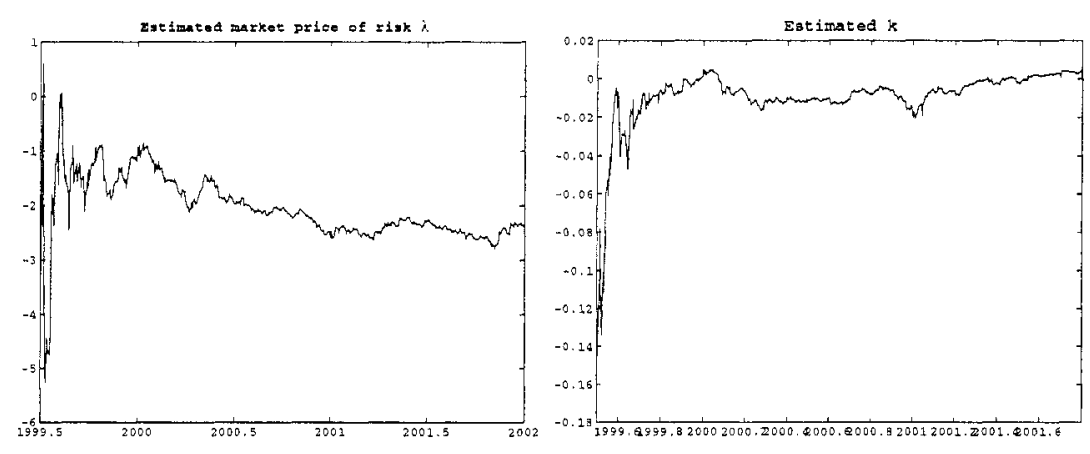

Figure 7. Estimated $\lambda$ and $k$
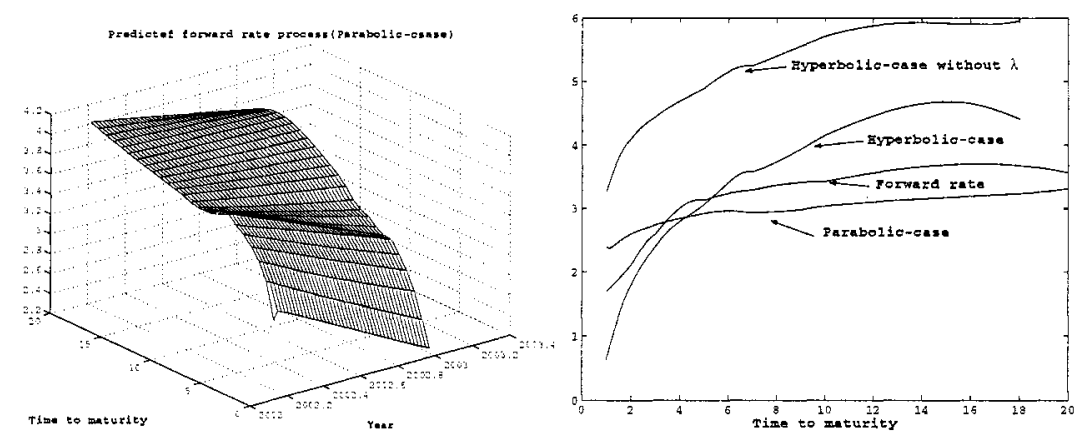

Figure 8. Predicted forward rate (Parabolic case) and true value

\section{Concluding remarks}

As shown in Fig.8, we could construct the parabolic type partial differential equation for the forward rate dynamics whose solution fits the future value of the forward rate better than the hyperbolic model. From the existence for the diffusion term, the shape of the predicted forward rate becomes flat and so the predicted forward rate for the parabolic case fits the real rate quite well. The calibration of the proposed model is very important to applying the mathematical algorithm to the practical situation and this should be done in the empirical probability rather than risk-neutral probability. For the identification problem in the parabolic case, we identified the term $k \frac{\partial^{2} f(t, x)}{\partial x^{2}}+\lambda \sqrt{q(x, x)}$ for the unknown $k$ and $\lambda$. From the obtained results, in the empirical probability world we need an extra term $k \frac{\partial^{2} f(t, x)}{\partial x^{2}}$ or $k \frac{\partial^{2} f(t, x)}{\partial x^{2}}+\lambda \sqrt{q(x, x)}$. The form of these terms are not theoretically derived and we only set the function form artificially. It 
should be noted that in the parabolic case, $\lambda=0$ does not mean the risk neutral world because we still have an extra term $k \frac{\partial^{2} f(t, x)}{\partial x^{2}}$.

Although Cont [5] proposed that the boundary value processes be stripped out of the original partial differential equation, we set the mixed boundary condition for the forward rate process. In our case, we can consider the more general type than Cont's model, e.g., the presence of boundary noises. If the covariance kernel is finite dimensional, we can transform the original measure to the risk neutral measure. If we do not consider the pricing of the future derivatives, it seems that this finite dimensionality condition is not required. However for the optimal portfolio problem with power utility we need this finite-dimensionality condition to support the optimal portfolio.

The most important part of this paper is how to fit the proposed model to the historical data. In the risk-neutral probability world, we only need to identify the kernel of the noise. However we can not reproduce the real future forward rate process from risk neutral case. The obtained empirical results strongly depend on the interpolation method which was used to convert the yield curve to forward rate process. In order to avoid this differential instability problem, we should reformulate the parameter identification problem as the filtering problem with discrete-time observation data. In such a reformulation, we need not to use the interpolation method and differentiation with respect to time-to-maturity variable.

\section{References}

[1] J. James and N. Webber. Interest Rate Modeling. John Willey \& Sons, Ltd, Chicheter New York, 2002.

[2] P. Santa-Clara and D. Sornette. The dynamics of the forward rate curve with stochastic string shocks. Review of Financial Studies, 14:149-185, 2001.

[3] S. Aihara and A. Bagchi. Stochastic hyperbolic model for infinite-dimensional forward rates and option pricing. Mathematical Finance, 15:27-47, 2005.

[4] J.P Bouchaud, N. Sagna, R. Cont, N.EL-Karoui, and M. Potters. Phenomenology of the interest rate curve. Applied Mathathematical Finance, 6:209-232, 1999.

[5] R. Cont. Modeling term structure dynamics: An infinite dimensional approach. Int. J. Theoretical and Applied Finace, 8:357-380, 2005.

[6] G. Da Prato and J. Zabczyk. Stochastic equations in infinite dimensions. Cambridge University Press, 1992.

[7] E. Pardoux. Stochastic partial differential equations and filtering of diffusion processes. Stochastics, 3:127-167, 1979. 